\title{
Inhibition of Hemozoin Formation in Plasmodium falciparum Trophozoite Extracts by Heme Analogs: Possible Implication in the Resistance to Malaria Conferred by the $\beta$-Thalassemia Trait
}

\author{
James A. Martiney,* Anthony Cerami,* \\ and Andrew F. G. Slater*t \\ *The Picower Institute for Medical Research, Manhasset, New York, U.S.A. \\ The Karolinksa Institute, Division of Toxicology, Stockholm, Sweden
}

\begin{abstract}
Background: Human falciparum malaria, caused by the intracellular protozoa Plasmodium falciparum, results in 1-2 million deaths per year. $P$. falciparum digests host erythrocyte hemoglobin within its food vacuole, resulting in the release of potentially toxic free heme. A parasite-specific heme polymerization activity detoxifies the free heme by cross-linking the heme monomers to form hemozoin or malaria pigment. This biochemical process is the target of the widely successful antimalarial drug chloroquine, which is rapidly losing its effectiveness due to the spread of chloroquine resistance. We have shown that chloroquine resistance is not due to changes in the overall catalytic activity of heme polymerization or its chloroquine sensitivity. Therefore, the heme polymerization activity remains a potential target for novel antimalarials. In this study, we investigated the ability of heme analogs to inhibit heme polymerization and parasite growth in erythrocytes.

Materials and Methods: Incorporation of radioactive hemin substrate into an insoluble hemozoin pellet was used to determine heme polymerization. Incorporation of radioactive hypoxanthine into the nucleic acid of di-
\end{abstract}

viding parasites was used to determine the effects of heme analogs on parasite growth. Microscopic and biochemical measurements were made to determine the extent of heme analog entry into infected erythrocytes. Results: The heme analogs tin protoporphyrin IX (SnPP), zinc protoporphyrin IX (ZnPP), and zinc deuteroporphyrin IX, 2,4 bisglycol (ZnBG) inhibited polymerization at micromolar concentrations (ZnPP $<<$ SnPP $<$ ZnBG). However, they did not inhibit parasite growth since they failed to gain access to the site of polymerization, the parasite's food vacuole. Finally, we observed high ZnPP levels in erythrocytes from two patients with $\beta$-thalassemia trait, which may inhibit heme polymerization.

Conclusions: The heme analogs tested were able to inhibit hemozoin formation in Plasmodium falciparum trophozite extracts. The increased ZnPP levels found in thalassemic erythrocytes suggest that these may contribute, at least in part, to the observed antimalarial protection conferred by the $\beta$-thalassemia trait. This finding may lead to the development of new forms of antimalarial therapy.

\section{INTRODUCTION}

Malaria remains a deadly disease in much of the developing world, with at least $\mathbf{3 0 0}$ million cases a year and 2 million deaths, mostly of children

Address correspondence and reprint requests to: James A. Martiney, The Picower Institute for Medical Research, 350 Community Drive, Manhasset, NY 11030, U.S.A. under 5 years of age. Human malaria is caused by intracellular protozoa of the genus Plasmodium, of which Plasmodium falciparum is by far the most lethal, accounting for more than $95 \%$ of all deaths and morbidity. The high number of people living in malaria-endemic regions of the world ( 2.5 billion) coupled with frequent mortality among children provide a powerful driving force for the genetic selection of protection against the disease. Some of the genetic abnor- 
malities associated with innate protection against malaria include sickle cell anemia, glucose-6phosphate dehydrogenase deficiency, the thalassemias, erythrocyte cytoskeletal and surface membrane abnormalities and other hemoglobinopathies (Hemoglobin C, E, and F) (reviewed in Ref. 1).

P. falciparum trophozoites obtain their amino acid requirements by degrading hemoglobin within an acidic food vacuole, a specialized organelle similar to mammalian lysosomes $(2,3)$. Heme is released during this process and converted to a nontoxic polymer (hemozoin or malaria pigment) by a parasite-specific heme polymerizing activity (4). A similar heme polymerization activity has also been detected in trophozoite extracts of the rodent malaria Plasmodium berghei (5). Although there is still some controversy as to its exact biochemical nature of the catalytic activity $(6-8)$, it is clear that this process is the target of the antimalarial drug chloroquine, until recently the most frequently used chemotherapeutic for malaria prophylaxis and cure.

For more than 45 years, chloroquine has been a very successful, widely used antimalarial drug, due to its specificity, effectiveness, stability, and safety (9). However, the spread of resistance to chloroquine, along with the development of resistance to other antimalarials such as mefloquine and quinine, call for an urgent need for new drugs. Chloroquine resistance cannot be explained by a mutation directly affecting the heme polymerization catalytic activity. The polymerizing activities isolated from trophozoite extracts of either chloroquine-resistant or -sensitive strains have similar overall activity and sensitivity to chloroquine and other antimalarials (Ref. 9 and A. F. G. Slater, personal communication). This activity is therefore a target for the development of novel drugs that would circumvent chloroquine resistance.

The aim of this study was to investigate the potential use of heme analogs to act as inhibitors of the trophozoite-derived heme polymerization process. The ability of these compounds to inhibit parasite growth in a erythrocyte culture system was also tested. Finally, since thalassemic erythrocytes contain higher levels of the $\mathrm{ZnPP}$ heme analog, we investigated the potential contribution of ZnPP-mediated heme polymerization inhibition to the antimalarial protection conferred by the $\beta$-thalassemia hemoglobinopathy.

\section{MATERIALS AND METHODS}

\section{Parasites}

Cultures of $P$. falciparum were grown in $\mathrm{A}^{+}$human erythrocyte suspensions using RPMI-1640 (GIBCO, Gaithersburg, MD, U.S.A.) medium supplemented with $25 \mathrm{mM}$ HEPES ( $\mathrm{pH} 7.35$ ), $0.2 \% \mathrm{NaHCO}_{3}(23 \mathrm{mM}), 0.2 \%$ (D)-glucose, and $10 \%$ human $\mathrm{A}^{+}$plasma, and maintained at $37^{\circ} \mathrm{C}$ in candle jars according to the method of Trager and Jensen (10-11). Parasites were stage synchronized by incubating mainly ring-stage infected erythrocytes in 5\% sorbitol for $10 \mathrm{~min}$ at room temperature (12). Cultures were allowed to undergo one growth cycle ( 2 days) before use. Strains used were the chloroquine-sensitive strain D10 and the chloroquine-resistant isolate Dd2 (kindly provided by Dr. Michael Lanzer, Sloan Kettering).

\section{Parasite Growth Measurements}

Parasite growth was monitored by measuring incorporation of $\left[{ }^{3} \mathrm{H}\right]$-hypoxanthine into the nucleic acids of the parasite as previously described (13). Effects of porphyrins on parasite growth was determined by measuring the reduction in radiolabel incorporation compared with controls. Briefly, assays were performed in 96-well microtitre plates, each well containing $200 \mu \mathrm{l}$ of synchronized ring-stage infected erythrocytes in complete media and $50 \mu \mathrm{l}$ of porphyrin dilution or solvent. The final hematocrit was $1.5 \%$, and the percentage parasitemias ranged from 1 to $5 \%$. After $24 \mathrm{hr}$ of candle jar incubation at $37^{\circ} \mathrm{C}$, $2.5 \mu \mathrm{Ci}$ of $\left[{ }^{3} \mathrm{H}\right]$-hypoxanthine $(17 \mathrm{Ci} / \mathrm{mmol} ; \mathrm{Am}-$ ersham, Arlington Heights, IL, U.S.A.) was added to each well. After $18 \mathrm{hr}$, the wells were harvested onto glass fiber filters, dried and placed in scintillation vials with $7 \mathrm{ml}$ of scintillation fluid. Vials were counted in a liquid Beckman LS7800 scintillation counter. Porphyrin half-maximal inhibition $\left(\mathrm{IC}_{50}\right)$ values were calculated by least squares curve fitting (Origin; Microcal Corp., Northampton, MA, U.S.A.). IC $_{50}$ values represent the molar concentration which decreases $\left[{ }^{3} \mathrm{H}\right]$-hypoxanthine incorporation by $50 \%$ compared with drug-free controls.

\section{Trophozoite Extract Preparation}

Heme polymerization activity was isolated as previously described (4). Briefly, synchronized parasite cultures were harvested by saponin lysis at the late trophozoite stage. Erythrocyte-free 
trophozoites were washed three times in PBS and stored at $-80^{\circ} \mathrm{C}$ for up to 3 months, with no detectable loss of polymerizing activity. The pellet was then homogenized on ice, immediately before the experiment, in PBS, $\mathrm{pH} 7.0$, with 1.5 $\mathrm{mM} \mathrm{MgCl}, 1 \mathrm{mM}$ PMSF, $1 \mathrm{mM} 1,10$ phenanthroline, $0.1 \mathrm{mM}$ leupeptin, and $50 \mu \mathrm{M}$ pepstatin, and mechanically disrupted with a Dounce homogenizer. The trophozoite homogenate was centrifuged at $4^{\circ} \mathrm{C}$ and $10,000 \times g$ for $30 \mathrm{~min}$, and the supernatant discarded. The membraneand hemozoin-rich pellet was resuspended in a small volume of PBS and immediately used in the polymerization assay.

\section{Heme Polymerization Assay}

Heme polymerization activity was assayed using $\left[{ }^{14} \mathrm{C}\right]$-hemin substrate essentially as described by Slater and Cerami (4). Briefly, trophozoite extracts were incubated at $37^{\circ} \mathrm{C}$ in $150 \mathrm{mM}$ phosphate buffer, $\mathrm{pH}$ 5.2, with $15 \mu \mathrm{m}$ substrate in the presence of different concentrations of inhibitor or solvent. Reactions were terminated after $8 \mathrm{hr}$ by the addition of $2 \%$ SDS in $0.1 \mathrm{M}$ sodium bicarbonate buffer, pH 9.5. The samples were then washed in bicarbonate buffer and incubated overnight in $1 \mathrm{mg} / \mathrm{ml}$ Protease E (Sigma Chemical Co., St. Louis, MO, U.S.A.), in $50 \mathrm{mM}$ TRIS $\mathrm{HCl}, \mathrm{pH}$ 7.5. The hemozoin pellet was recovered by centrifugation and the amount of heme incorporated determined by scintillation counting in a Beckmann LS7800.

\section{Determination of Protoporphyrin Concentrations}

All porphyrin measurements were done using a derivation of Piomelli's method for Free Erythrocyte Porphyrin (FEP) measurement from blood $(14,15)$. Briefly, 20-50 $\mu$ l protoporphyrin was added to $2 \mathrm{ml}$ of a $4: 1$ mixture of ethyl acetate/ acetic acid and vortexed for $10 \mathrm{~min}$. The suspension is mixed with $2 \mathrm{ml}$ of $1.5 \mathrm{~N} \mathrm{HCl}$ by inversion. The mixture is centrifuged for $3 \mathrm{~min}$ at 2500 $\mathrm{rpm}$, room temperature. The resulting lower $\mathrm{HCl}$ phase was read in a fluorescence spectrophotometer against a $1.5 \mathrm{~N} \mathrm{HCl}$ blank (excitation at 405 , emission peak at 596). A $0.050 \mu \mathrm{g} / \mathrm{ml}$ coproporphyrin III (CPP III) standard (Porphyrin Products, Logan, UT, U.S.A.) was used to calibrate the luminiscence spectrometer (LS50 B, Perkin Elmer). The concentration of FEP was calculated from the formula:
FEP $\mu \mathrm{g} / \mathrm{ml}$ sample $=(F x) *(C s / F s) *(\mathrm{HCl}$ final volume $/$ volume sample used) $*(1 / 1.11)$

where $F x, F s=$ fluorescence of unknown and standard; $C s=$ concentration of standard $(\mu \mathrm{g} /$ $\mathrm{ml}) ; 1.11=$ value used to convert fluorescence of porphyrins in the sample to fluorescence of CPP III in $1.5 \mathrm{~N} \mathrm{HCl}$. CPP III concentration in the standard was calculated directly from the absorbance at $\mathrm{OD}_{401}$, where 0.667 corresponds to 1 $\mu \mathrm{g} / \mathrm{ml}$. There was a linear relationship between fluorescence and CPP III concentration (measured by absorbance) up to $0.7 \mu \mathrm{g} / \mathrm{ml}$. Therefore, unknown samples were diluted accordingly to fall within the linear range of the standard calibration. Erythrocyte FEP samples were measured using the same protocol and the results normalized for percentage hematocrit to yield FEP $\mu \mathrm{g} / \mathrm{ml}$ of blood. The spectroscope was a HewlettPackard 8450A.

\section{Microscopy}

Thin blood smears were immersed in methyl alcohol followed by staining with Giemsa (Fisher Diagnostics, Orangetown, NY, U.S.A.) for visualization of parasites. Light and polarized light microscopy was done using an Olympus $\mathrm{BH} 2$ microscope.

\section{Reagents}

Ferriprotoporphyrin IX chloride (hemin) and coproporphyrin I dihydrochloride were purchased from Aldrich (Milwaukee, WI, U.S.A.). Coproporphyrin III, protoporphyrin IX dimethyl ester, uroporphyrin III dihydrochloride, zinc protoporphyrin IX, and zinc deuteroporphyrin IX, 2,4 Bis glycol were purchased from Porphyrin Products Inc. (Logan, UT, U.S.A.). Stock solutions of $2 \mathrm{mM}$ hematin in $0.01 \mathrm{~N} \mathrm{NaOH}$ were prepared fresh and the concentration measured using the pyridine hemochrome method (16). Briefly, the heme is solubilized in $\mathrm{NaOH}$, and an aliquot reacted with pyridine and $\mathrm{K}_{3} \mathrm{Fe}(\mathrm{CN})_{6}$ and read in a spectrophotometer. The heme is then reduced with sodium hydrosulfite and spectroscopically read again. The sample concentration is then calculated by substracting oxidized from reduced heme absorptions and dividing by a millimolar extinction coefficient of 20.7. Three milliliters of this stock was added to $1 \mu \mathrm{Ci}\left[{ }^{14} \mathrm{C}\right]$ hemin chloride from Leeds Radioporphyrins (Leeds Radioporphyrins, United Kingdom). Chloroquine was purchased from Sigma. All sol- 
vents and reagents were of the highest grade available.

\section{Blood Samples}

Blood samples from two individuals classified as having $\beta$-thalassemia minor (or $\beta$-thalassemia trait) were obtained from the Clinic of Pediatric Hematology at New York Hospital (New York, NY, U.S.A.).

\section{RESULTS}

\section{Heme Polymerization Can Be Inhibited in Vitro by Heme Analogs}

Heme polymerization involves the cross-linking of the iron of one heme to a carboxylate group of another $(16,17)$. A change in the central metal moiety could inhibit the polymerization process. Zinc protoporphyrin IX (ZnPP) (Fig. 1) is a naturally occurring metalloporphyrin, used clinically to inhibit neonatal heme catabolism (18). We assessed the inhibitory effect of this noniron heme analog on trophozoite-catalyzed heme polymerization (Fig. 2). As predicted, ZnPP inhibited polymerization, but only at relatively high concentrations (average $\mathrm{IC}_{50}$ of $480 \pm 281 \mu \mathrm{M}$, $n=7$ ). There was no significant difference in the sensitivity to ZnPP inhibition of polymerization between chloroquine-resistant and -sensitive trophozoites extracts (Fig. 3). To determine the nature of the inhibition, trophozoite extracts were incubated in the presence of increasing ZnPP concentrations at five different heme substrate concentrations. A Lineweaver-Burk reciprocal plot of the data demonstrates complex inhibitory kinetics (Fig. 4). A competitive component is apparent at low inhibitor concentrations $(0-250$ $\mu \mathrm{M} \mathrm{ZnPP}$ ) and a noncompetitive component evident at higher concentrations.

The second heme analog tested was tin protoporphyrin (SnPP) (Fig. 1), a strong competitive inhibitor of human heme oxygenase and a pharmacological agent administered to newborns with severe hyperbilirubinemia (19). SnPP was solubilized in a $1: 195 \%$ ethanol/0.1 N NaOH v/v, which provides a 6-fold greater solubility than ZnPP in $20 \%$ methanol when used in our aqueous assay conditions (data not shown). When tested in the heme polymerization assay, SnPP inhibited polymerization with an average $\mathrm{IC}_{50}$ of $75 \pm 20 \mu \mathrm{M}$, a 7-fold increase over ZnPP (Fig. 5). In comparison, the $\mathrm{IC}_{50}$ for chloroquine in this system is approxi-

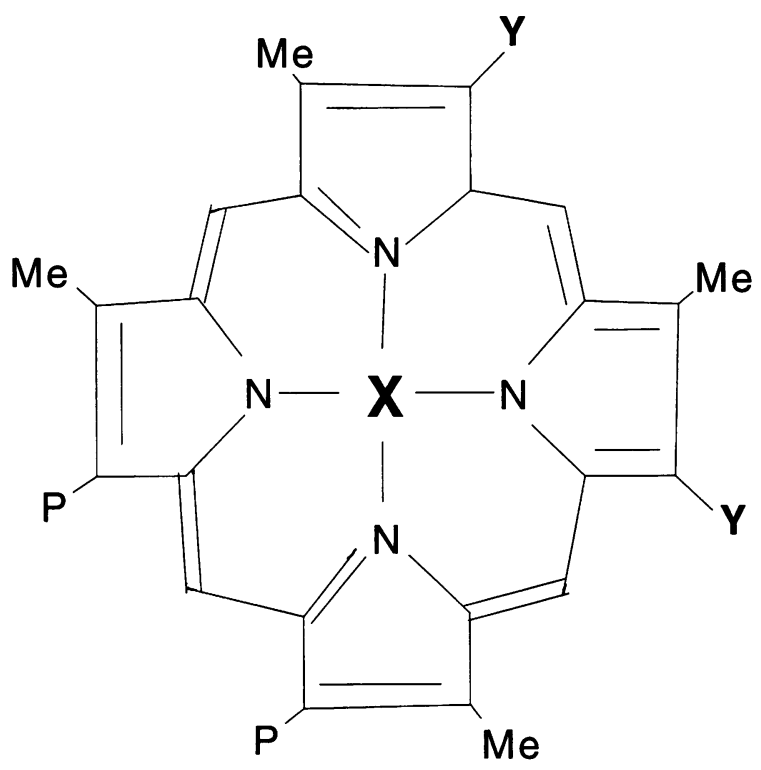

FIG. 1. Chemical structures of heme polymerization inhibitors as compared to heme

\begin{tabular}{c|c|c|l|l} 
& $\begin{array}{l}\text { Iron Protoporphyrin } \\
\mathrm{IX} \text { (heme) }\end{array}$ & $\begin{array}{l}\text { Zinc Protoporphyrin } \\
\mathrm{IX}(\mathrm{ZnPP})\end{array}$ & $\begin{array}{l}\text { Zinc Protoporphyrin } \\
\mathrm{IX} \mathrm{2,4} \mathrm{Bisglycol} \\
(\mathrm{ZnBG})\end{array}$ & $\begin{array}{l}\text { Tin Protoporphyrin } \\
\mathrm{IX}(\mathrm{SnPP})\end{array}$ \\
\hline $\mathbf{X}$ & $\mathrm{Fe}$ & $\mathrm{Zn}$ & $\mathrm{Zn}$ & $\mathrm{Sn}$ \\
\hline $\mathbf{Y}$ & $-\mathrm{CH}=\mathrm{CH}_{2}$ & $-\mathrm{CH}=\mathrm{CH}_{2}$ & $-\mathrm{CHOHCH} \mathrm{HH}_{2}$ & $-\mathrm{CH}=\mathrm{CH}_{2}$ \\
\hline $\mathbf{M e}$ & $-\mathrm{CH}_{3}$ & $-\mathrm{CH}_{3}$ & $-\mathrm{CH}_{3}$ & $-\mathrm{CH}_{3}$ \\
\hline $\mathbf{P}$ & $-\mathrm{CH}_{2}-\mathrm{CH}_{2}-\mathrm{COOH}$ & $-\mathrm{CH}_{2}-\mathrm{CH}_{2}-\mathrm{COOH}$ & $-\mathrm{CH}_{2}-\mathrm{CH}_{2}-\mathrm{COOH}$ & $-\mathrm{CH}_{2}-\mathrm{CH}_{2}-\mathrm{COOH}$ \\
\hline
\end{tabular}

Hemozoin is formed upon the chemical crosslinking of the central iron of one heme with a carboxy group of another heme. All heme polymerization inhibitors contained the carboxy group as part of the propionate side chain $(\mathrm{P})$. The central metal $(\mathrm{X})$ varies according to the inhibitor, probably serving as chain terminators. The vinyl groups $(\mathrm{Y})$ remain the same, except in ZnBG, in which they were substituted for glycols, enhancing compound solubility. Methyl groups (Me) remain unchanged throughout.

mately $50 \mu \mathrm{M}$ (data not shown). Similarly to ZnPP, a Lineweaver-Burk reciprocal plot of polymerization in the presence of increasing amounts of inhibitor demonstrated complex inhibitory kinetics, with competitive and noncompetitive components at lower and high inhibitor concentrations, respectively (data not shown).

In order to obtain even better solubility, and thus decrease potential toxicity, another heme oxygenase inhibitor, zinc deuteroporphyrin IX, 2,4 Bis glycol ( $\mathrm{ZnBG}$ ) was used. ZnBG inhibited heme oxygenase with a $k_{i}$ of $0.003 \mu \mathrm{M}$, indicating that it is 10 times more potent than SnPP (20). This heme analog is also less photoreactive in vitro and lacks the in vivo photosensitizing capabilities of SnPP. Finally, it was shown not to 


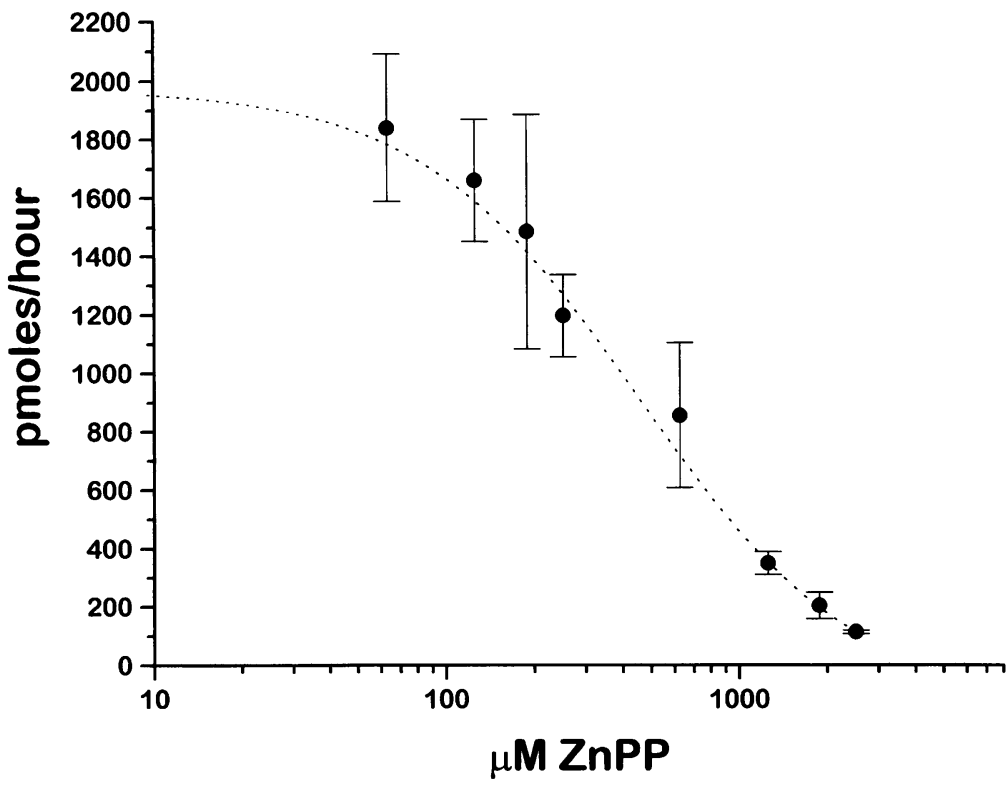

FIG. 2. Inhibition of trophozoite extract-derived heme polymerization activity by the heme analog ZnPP

Parasites were cultured at increasing ZnPP concentrations. Data are expressed as incorporation of radioactive heme into an insoluble polymer (hemozoin). Halfmaximal inhibitory effect $\left(\mathrm{IC}_{50}\right)$ of $\mathrm{ZnPP}$ is observed at a concentration of $\approx 500$ $\mu \mathrm{M}$. The line represents the nonlinear least squares fit to the data. Each data point represents mean \pm standard errors of two data points from a representative experiment. produce any side effects when it was administered to neonates with iatrogenic hemolysis (21). The addition of glycol groups enhanced solubility in our aqueous assay 2- to 3-fold over SnPP (data not shown). ZnBG inhibited trophozoite extractcatalyzed heme polymerization with an $\mathrm{IC}_{50}$ of $3.2 \pm 0.5 \mu \mathrm{M}, n=4$, (Fig. 6).

\section{Heme Analogs Do Not Inhibit Parasite Growth and Maturation}

We tested ZnPP, ZnBG, and other porphyrins (coproporphyrin III, protoporphyrin IX, uropor- phyrin III) for their ability to inhibit $P$. falciparum intraerythrocytic growth and maturation. Porphyrins have very low aqueous solubility; solvents compatible with our assay include ethanol, methanol, DMSO, ethanolamine, and $\mathrm{NaOH}$, which inhibit parasite growth in cultures at concentrations above $2.0 \% \mathrm{v} / \mathrm{v}$ for methanol and ethanol, and $0.02 \% \mathrm{v} / \mathrm{v}$ for all others (data not shown). We therefore used the highest porphyrin concentrations possible without inducing toxicity by the solvent. No inhibition of parasite growth by $\mathrm{ZnBG}$, the most water soluble heme

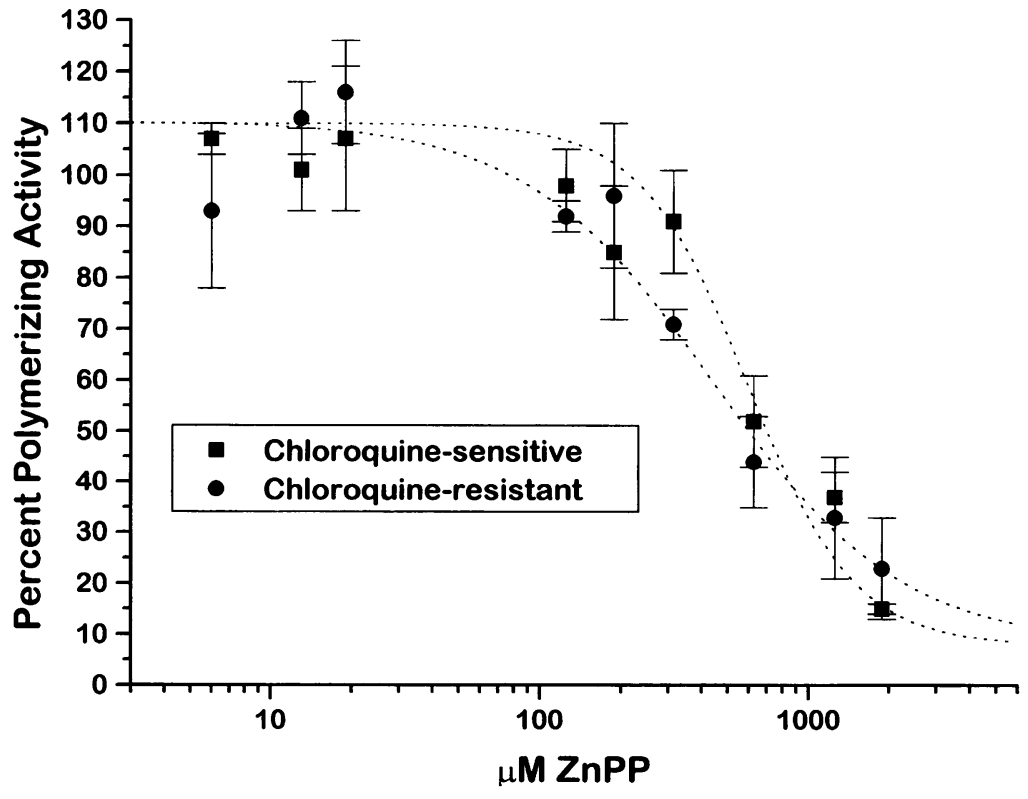

FIG. 3. Inhibition of heme polymerization by ZnPP in trophozoite extracts from chloroquine-resistant and -sensitive strains

Data are expressed as the percentage of polymerization activity compared with drug-free controls. The $\mathrm{IC}_{50}$ for the chloroquine-sensitive strain is $698 \pm$ $83 \mu \mathrm{M}$, and $604 \pm 74 \mu \mathrm{M}$ for the chloroquine-resistant strain. Chloroquineresistant data is shown as closed circles, chloroquine-sensitive as closed squares. The lines represent the nonlinear least squares fit to the data of a representative experiment. 


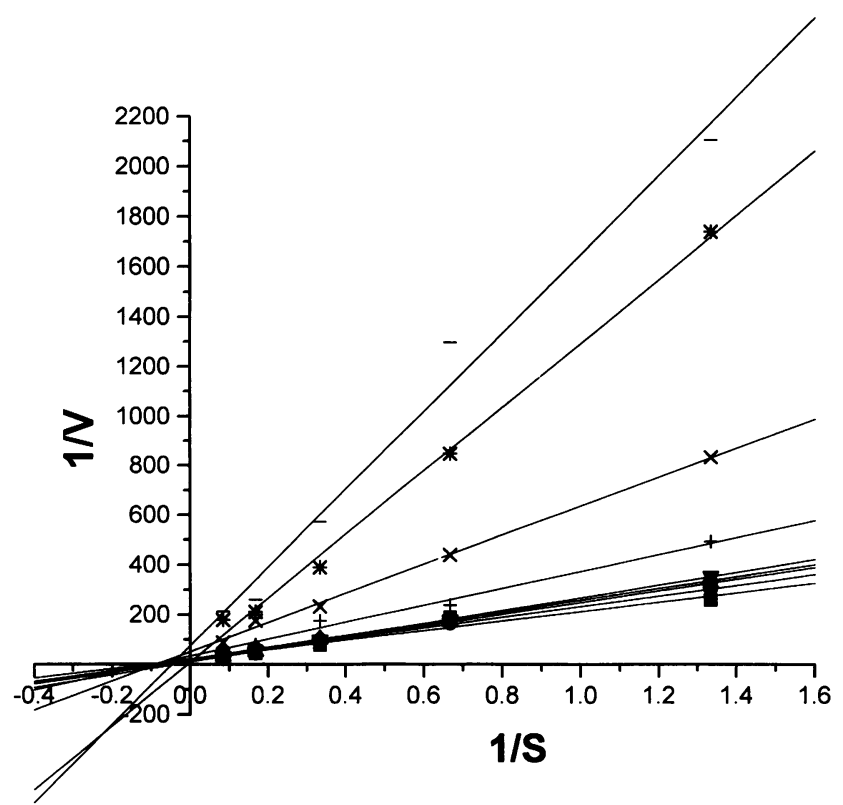

analog, was detected at concentrations 30-fold higher than required for inhibition of heme polymerization (Table 1). Similarly, none of the other porphyrins tested demonstrated any inhibitory activity. When $P$. falciparum cultures were incubated with $\mathrm{ZnBG}$ and chloroquine concomitantly, the resulting $\mathrm{IC}_{50}$ was dependent solely on the chloroquine sensitivity (data not shown).

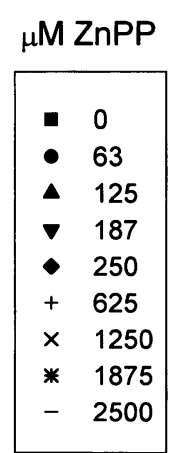

FIG. 4. Lineweaver-Burk reciprocal plot of heme polymerization inhibition by various concentrations of ZnPP

Velocity is expressed as picomoles of heme incorporated into hemozoin per hour. Reciprocal velocity (1/V) units are $10^{-5}$ pmoles/hr. Reciprocal substrate $(1 / \mathrm{S})$ is expressed as $\mu \mathrm{M}$ heme $^{-1}$. Representative experiment of $n=3$. The lines represent the linear fit to the data.

\section{Heme Analogs Do Not Gain Access to the Parasite's Polymerizing Compartment}

We suspected that the physicochemical properties of heme analogs would prevent their access to the site of heme polymerization, the food vacuole. Once inside the erythrocyte cytoplasm, compounds gain access to the food vacuole dur-

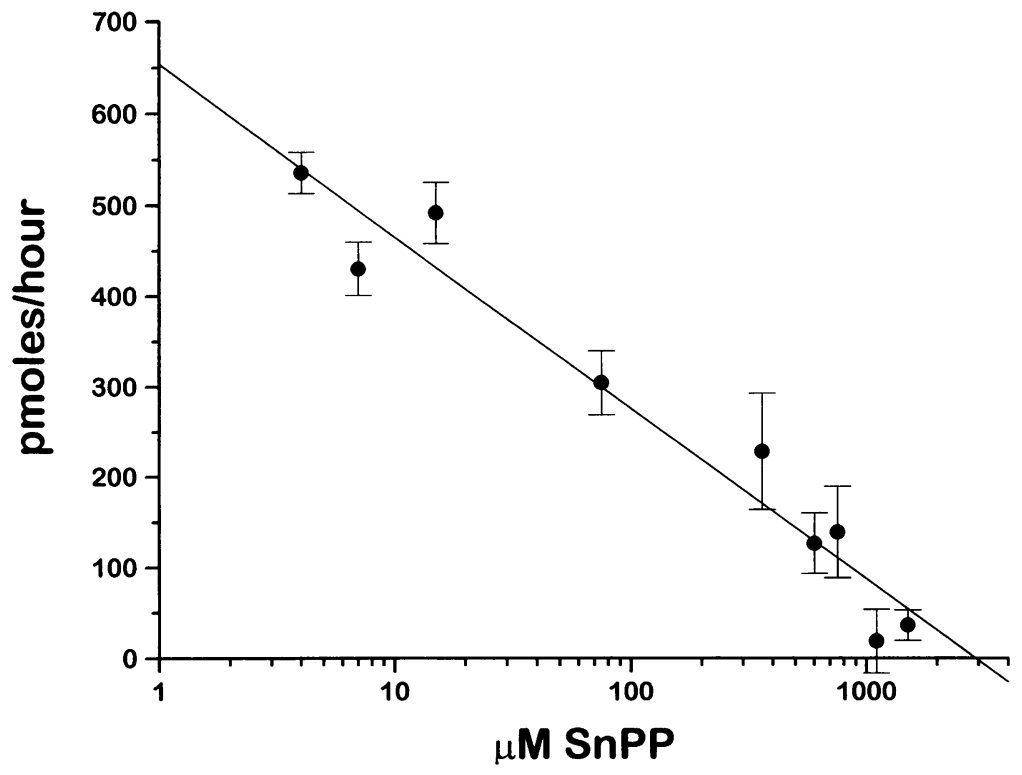

FIG. 5. Inhibition of trophozoite extract-derived heme polymerization activity by SnPP

Data are expressed as picomoles of radioactive heme incorporated into an insoluble polymer (hemozoin) per hour in the presence of increasing amounts of SnPP. The line represents the linear fit to the data. Each data point represents mean \pm standard errors of two data points from a representative experiment of $n=5$. 


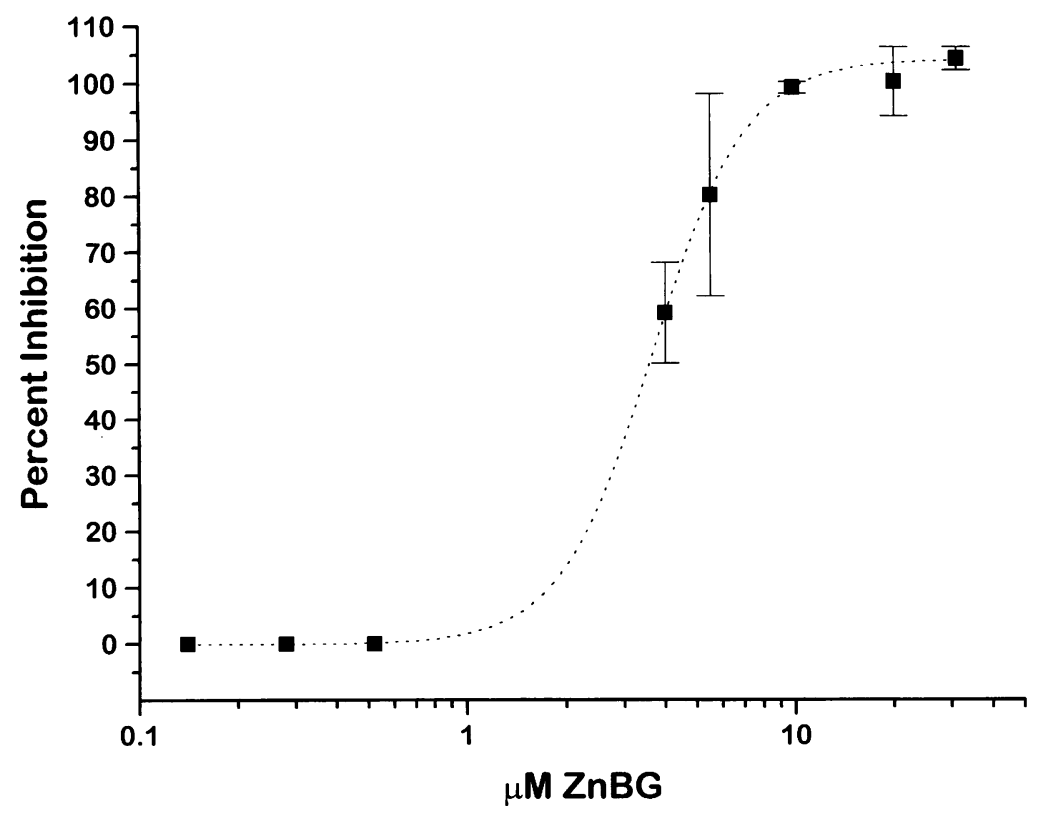

FIG. 6. Inhibition of trophozoite extract derived heme polymerization by ZnBG

Data are expressed as incorporation of radioactive heme into an insoluble polymer (hemozoin) in the presence of increasing amounts of $\mathrm{ZnBG}$. The line represents the non-linear least squares fit to the data. The $\mathrm{IC}_{50}$ for this particular experiment is $3.6 \mu \mathrm{M}$. Each data point represents mean \pm standard errors of two data points from a representative experiment of $n=4$. ing hemoglobin intake by the parasite. In addition, some large compounds are able to gain direct access to the parasite cytoplasm from serum via a putative parasitophorous duct (22). We measured ZnBG uptake into infected erythrocytes by observing blood smears from cultures treated at various time points with $\mathrm{ZnBG}$ by light microscopy under Nomarski optics and polarized light. ZnBG was visible on the smears as aggregates and precipitates, but it was not detected inside the erythrocytes for up to $17 \mathrm{hr}$ following its addition (data not shown). Finally, when ZnBG uptake into infected erythrocytes was measured using the FEP method, $\leq 1 \%$ ZnBG was detected inside infected erythrocytes after 24 $\mathrm{hr}$, and $\leq 2 \%$ after $48 \mathrm{hr}$ (Table 2 ). In summary, our results demonstrate that $\mathrm{ZnBG}$ does not permeate the infected erythrocyte membrane or gain direct access to the parasite, thereby explaining its failure to inhibit parasite growth.

\section{The High Intraerythrocytic ZnPP Levels Present in Thalassemic Erythrocytes Could Contribute to the Associated Protection to Malaria}

A "natural" situation in which high ZnPP levels are present inside erythrocytes is thalassemia. It has been documented that thalassemic erythrocytes contain higher levels of ZnPP than normal erythrocytes $(23,24)$. The correlation between the geographical distribution of malaria and the

TABLE 1. Inhibition of in vitro parasite growth by porphyrins

\begin{tabular}{llc}
\hline Compound & \multicolumn{1}{c}{ Solvent } & No Inhibition at: \\
\hline Zinc deuteroporphyrin bisglycol & $2 \mathrm{mM} \mathrm{NaOH} / 2 \% \mathrm{EtOH}$ & $\leq 104.8 \mu \mathrm{M}$ \\
Zinc deuteroporphyrin bisglycol & $0.02 \%$ ethanolamine & $\leq 69.4 \mu \mathrm{M}$ \\
Zinc protoporphyrin IX & $2 \mathrm{mM} \mathrm{NaOH} / 2 \% \mathrm{EtOH}$ & $\leq 2.6 \mu \mathrm{M}$ \\
Zinc protoporphyrin IX & $0.02 \%$ ethanolamine & $\leq 5.2 \mu \mathrm{M}$ \\
Coproporphyrin III & $2 \mathrm{mM} \mathrm{NaOH} / 2 \% \mathrm{EtOH}$ & $\leq 134.4 \mu \mathrm{M}$ \\
Coproporphyrin III & $0.02 \%$ ethanolamine & $\leq 165.0 \mu \mathrm{M}$ \\
Protoporphyrin IX & $2 \mathrm{mM} \mathrm{NaOH} / 2 \% \mathrm{EtOH}$ & $\leq 0.8 \mu \mathrm{M}$ \\
Uroporphyrin III & $2 \mathrm{mM} \mathrm{NaOH} / 2 \% \mathrm{EtOH}$ & $\leq 157.0 \mu \mathrm{M}$
\end{tabular}


TABLE 2. Infected erythrocytes are not permeable to $\mathrm{ZnBG}$

\begin{tabular}{lcc}
\hline $\begin{array}{l}\text { Conditions } \\
(+\mathbf{1 0 0} \boldsymbol{\mu M}\end{array}$ & $\begin{array}{c}\text { Recovery of } \\
\text { ZnBG at 24 hr } \\
\text { by [FEP] } \\
\text { ZnBG) }\end{array}$ & $\begin{array}{c}\text { Recovery of } \\
\text { ZnBG at 48 hr } \\
\text { betermination [FEP] } \\
\text { Determination }\end{array}$ \\
\hline Media alone & $112 \mu \mathrm{M}$ & $105 \mu \mathrm{M}$ \\
Media with RBC & $105 \mu \mathrm{M}$ & $97 \mu \mathrm{M}$ \\
RBC pellet & $0.8 \mu \mathrm{M}$ & $1.8 \mu \mathrm{M}$ \\
\hline
\end{tabular}

human thalassemias is also well documented with genetic support for the natural selection of thalassemias due to malaria pressure $(25,26)$. The molecular mechanism(s) underlying reduced malaria susceptibility in thalassemic individuals is, however, unclear. We propose that a component of this mechanism may involve the inhibition of heme detoxification via an increase in intracellular ZnPP levels.

We obtained two samples of blood from patients with $\beta$-thalassemia trait. Parasite growth in thalassemic erythrocytes was lower compared with age- and type-matched normal erythrocytes (Table 3). We then measured ZnPP content using our FEP determination assay. Our results mirror those of previous investigators, showing that thalassemic erythrocytes contain about a 2 -fold higher concentration of ZnPP than normal erythrocytes (Table 4 ). The correlation between the higher ZnPP levels in $\beta$-thalassemic erythrocytes

TABLE 3. $P$. falciparum growth in thalassemic erythrocytes as compared to age- and type-matched normal erythrocytes

\begin{tabular}{cccc}
\hline Experiment & $\begin{array}{c}\text { Normal } \\
\text { RBC }\end{array}$ & $\begin{array}{c}\text { Thalassemic } \\
\text { RBC }\end{array}$ & $\begin{array}{c}\text { \% } \\
\text { Reduction }\end{array}$ \\
\hline 1 & 62,757 & 15,126 & 76 \\
2 & 15,492 & 2,952 & 81 \\
3 & 21,925 & 4,690 & 79 \\
4 & 32,393 & 8,378 & 74 \\
Average & & \multicolumn{2}{c}{$77.5 \pm 3.1$} \\
\hline
\end{tabular}

Data are expressed as amount of radioactive hypoxanthine incorporated into actively dividing parasites, normalized to same hematocrit and parasitemia.
TABLE 4. FEP content in normal versus thalassemic $A^{+}$erythrocytes

\begin{tabular}{cccc}
\hline Experiment & $\begin{array}{c}\text { Normal } \\
\text { RBC }\end{array}$ & $\begin{array}{c}\text { Thalassemic } \\
\text { RBC }\end{array}$ & $\begin{array}{c}\text { Fold } \\
\text { Reduction }\end{array}$ \\
\hline 1 & $0.6 \mu \mathrm{M}$ & $1.5 \mu \mathrm{M}$ & 2.5 \\
2 & $0.8 \mu \mathrm{M}$ & $1.5 \mu \mathrm{M}$ & 1.9 \\
Average & & & $2.2 \pm 0.4$ \\
\hline
\end{tabular}

and the lower parasite growth suggests that ZnPP activity could inhibit heme polymerization.

\section{DISCUSSION}

$P$. falciparum malaria has been in the increase for the past 20 years, mostly due to the development of resistance against chloroquine. The mechanism of action of chloroquine, and possibly other aminoquinolines, is the heme polymerization process (4). This unique biochemical pathway renders the heme liberated during hemoglobin digestion nontoxic by cross-linking heme into an insoluble polymer (hemozoin) $(4,16)$. The heme polymerization activity extracted from either chloroquine-sensitive and -resistant trophozoites demonstrated no difference in sensitivity to chloroquine (9). Kinetic analyses of chloroquine inhibitory profiles also showed very similar $k_{i}$ 's using heme polymerization activity from chloroquine-resistant and -sensitive $P$. falciparum trophozoites (A. F. G. Slater, personal communication). Chloroquine resistance is not associated with a decrease in the sensitivity of polymerization activity to inhibition by quinoline-based drugs. The heme polymerization activity is therefore a valid target for the development of novel antimalarial chemotherapeutics which could circumvent chloroquine resistance. We sought, therefore, to identify novel inhibitors of this critical, parasite-specific biochemical process.

Polymerization of heme involves an ironcarboxylate bond (16). We predicted that substitution of the central metal moiety would inhibit heme polymerization. Noniron heme analogs such as tin and zinc porphyrins have been developed as potent inhibitors of mammalian heme oxygenase, the rate-limiting enzyme in the heme degradative pathway. This enzyme utilizes heme (ferriprotoporphyrin IX) as substrate, cleaving the cyclical structure via a coupled oxidation reaction 
forming biliverdin IX, which is further reduced to bilirubin and eventually excreted in the bile. The main clinical use of these metalloporphyrins is in decreasing bilirubin formation in a variety of neonatal jaundice conditions such as hyperbilirubinemia $(27,28)$.

Metalloporphyrins are relatively insoluble in aqueous solutions. We therefore had to find solvent conditions that would be compatible with our polymerization assay system. Unfortunately, the best metalloporphyrin solvent system, having a basic $\mathrm{pH}$, was incompatible with the heme polymerization reaction, which has an acidic activity optimum (4). We therefore developed a combination of alcohols and small amounts of $\mathrm{NaOH}$ for metalloporphyrin solubilization which did not alter the heme polymerization reaction.

Iron-substituted porphyrins inhibited $P$. falciparum trophozoite heme polymerization activity: ZnPP was the least potent inhibitor; SnPP had an $\mathrm{IC}_{50}$ similar to chloroquine in our system; and ZnBG had an even better inhibitory activity. The inhibitory kinetics are complex, with a competitive component apparently evident at low inhibitor concentrations, but at higher concentrations a noncompetitive component is evident. Resolution of this issue requires a more clearly defined system than a crude trophozoite extract. We then tested these and other heme analogs for potential in vitro $P$. falciparum growth inhibitory activity. A wide range of heme analogs tested, including $\mathrm{ZnBG}$, failed to inhibit $P$. falciparum growth, even at concentrations that would result in significant in vitro inhibition of heme polymerization.

Intraerythrocytic stages of $P$. falciparum mature within the parasitophorous vacuole membrane, which forms from the host plasma membrane during invasion (29). Hemoglobin digestion and heme polymerization occur within the food vacuole, a low $\mathrm{pH}$ organelle not unlike mammalian lysosomes $(3,30,31)$. Compounds can enter the food vacuole in the following ways: (i) weak bases, such as chloroquine, accumulating in their protonated form due to the weak base effect; (ii) small hydrophobic molecules passively diffusing across the three membrane systems between the serum and the food vacuole; and (iii) molecules that gain access to the red blood cell cytoplasm being passively transported from the cytoplasm into the food vacuole during hemoglobin ingestion. In addition, there is controversial evidence for a putative parasitophorous duct which directly connects the parasite cytoplasm to the external medium $(32,33)$.

We interpreted the failure of heme analogs to inhibit parasite growth to their inability to gain access to the food vacuole. We studied the fate of added $\mathrm{ZnBG}$ to determine the site where entry into the vacuole was blocked. Based on light microscopy and FEP determination, we concluded that ZnBG can neither cross the erythrocyte membrane and accumulate in its cytoplasm, nor traverse any putative ducts directly into the parasite. A better delivery system, using lyophilized liposomes formulations of metalloproteins, could circumvent this blockage (34).

Finally, we investigated a natural situation in which a heme analog, $\mathrm{ZnPP}$, is found at high levels in the erythrocyte cytoplasm: $\beta$-thalassemia erythrocytes $(23,24)$. We first measured growth of $P$. falciparum in thalassemic erythrocytes compared with age- and type-matched normal erythrocytes. In four separate experiments, thalassemic erythrocytes inhibited $P$. falciparum growth by an average of $78 \pm 3 \%$. There has been some controversy as to the effect of the thalassemic erythrocyte environment on $P$. falciparum invasion, growth, and development. Some investigators have found no changes in parasite development in thalassemic erythrocytes $(35,36)$, or normal growth but enhanced susceptibility to oxidants (37), while other groups have seen inhibition of growth (38). In our system, we have measured a reproducible, constant decrease in parasite growth in thalassemic erythrocytes.

We also measured thalassemic intraerythrocytic ZnPP levels which were higher than normal. The intraerythrocytic ZnPP could gain access into the food vacuole, as it is associated with heme. In this way, as heme is the substrate for hemozoin formation, the presence of a ZnPPcontaining heme could inhibit heme polymerization activity. Several mechanisms have been proposed to explain the molecular basis of protection against malaria conferred by $\beta$-thalassemia. These include host erythrocyte biochemical abnormalities (1), high hemoglobin F content (38), iron deficiency (39), enhanced immune recognition (40), and impaired rosette formation (41). We would like to propose another protective factor, mainly that the elevated thalassemic intraerythrocytic ZnPP levels could inhibit heme polymerization. This is an area of investigation that we feel warrants more attention.

The geographical spread of chloroquine-resistance, the advent of resistance to other antimalarials such as mefloquine, quinine, and pyrimethamine, concomitant with the limited success of vaccines, make it imperative to develop new antimalarials. Ideally, such a compound would be 
parasite specific and able to circumvent the resistance to other known drugs. We have shown that chloroquine resistance is not a result of altered heme polymerization activity. Therefore, heme polymerization remains a highly vulnerable and indispensable biochemical pathway for the intraerythrocytic $P$. falciparum trophozoite which may be targeted for therapeutic intervention.

In conclusion, our experiments show that hemozoin formation can be exploited with respect to (i) the development of novel, substratebased competitive inhibitors of heme polymerization and (ii) the identification of nonquinoline molecules capable of coordination with the heme substrate, possibly providing a new class of antimalarial agents.

\section{ACKNOWLEDGMENTS}

We want to thank Gary Latortue for technical help and Dr. Patricia Griadino at the Pediatric Hematology Department of New York Hospital for the $\beta$-thalassemia blood samples. This work was supported by National Institutes of Health Grant RO1-AI-30660-04, Minority Individual in Postdoctoral Training Supplement Grant RO1AI-30660-S1 (JAM) and the UNDP/World Bank/ WHO Special Program for Research in Tropical Diseases (AFGS).

\section{REFERENCES}

1. Nagel RL, Roth Jr EF. (1989) Malaria and red cell genetic defects. Blood 74: 1213-1221.

2. Gluzman IY, Francis SE, Oksman A, Smith CE, Duffin KL, Goldberg DE. (1994) Order and specificity of the Plasmodium falciparum hemoglobin degradation pathway. J. Clin. Invest. 93: 1602.

3. Olliaro PL, Goldberg DE. (1995) The Plasmodium digestive vacuole: Metabolic headquarters and choice drug target. Parasitol. Today 11: 294.

4. Slater AFG, Cerami A. (1992) Inhibition by chloroquine of a novel haem polymerase enzyme activity in malaria trophozoites. Nature 355: 167.

5. Chou AC, Fitch CD. (1992) Heme polymerase: Modulation by chloroquine treatment of a rodent malaria. Life Sci. 51: 2073.

6. Warhurst DC. (1995) Haemozoin and the mode of action of blood schizontocides: More controversy. Parasitol. Today 11: 204.
7. Dorn A, Stoffel R, Matile H, Bubendorf A, Ridley RG. (1995) Malarial haemozoin/betahaematin supports haem polymerization in the absence of protein. Nature 374: 269.

8. Bendrat K, Berger BJ, Cerami A. (1995) Haem polymerization in malaria. Nature 378: 138.

9. Slater AFG. (1993) Chloroquine: Mechanism of drug action and resistance in Plasmodium falciparum. Pharmacol. Ther. 57: 203.

10. Trager W, Jensen JB. (1976) Human malaria parasites in continuous culture. Science 193: 673.

11. Jensen JB, Trager W. (1977) Plasmodium falciparum in culture: Use of outdated erythrocytes and description of the candle jar method. J. Parasitol. 63: 883.

12. Lambros C, Vanderberg JP. (1979) Synchronization of Plasmodium falciparum erythrocytic stages in culture. J. Parasitol. 65: 418.

13. Desjardins RE, Canfield CJ, Haynes JD, Chulay JD. (1979) Quantitative assessment of antimalarial activity in vitro by a semiautomated microdilution technique. Antimicrob. Agents Chemother. 16: 710.

14. Piomelli S. (1972) A micromethod for free erythrocyte porphyrins: The FEP test. J. Lab. Clin. Med. 81: 932.

15. Piomelli S, Brickman A, Carlos E. (1976) Rapid diagnosis of iron deficiency by measurement of free erythrocyte porphyrins and hemoglobin: The FEP/Hemoglobin ratio. Pediatrics 57: 136.

16. Slater AFG, Swiggard WJ, Orton BR, et al. (1991) An iron-carboxylate bond links the heme units of malaria pigment. Proc. Natl. Acad. Sci. U.S.A. 88: 325.

17. Slater AFG. (1992) Malaria pigment. Exp. Parasitol. 74: 362.

18. Rodgers PA, Vreman HJ, Stevenson DK. (1990) Heme catabolism in rhesus neonates inhibited by zinc protoporphyrin. Dev. Pharmacol. Ther. 14: 216.

19. Kappas A, Drummond GS, Manola T, Petmezaki S, Valaes T. (1988) Sn-protoporphyrin use in the management of hyperbilirubinemia in term newborns with direct Coombs-positive ABO incompatibility. Pediatrics 81: 485-497.

20. Vreman HJ, Lee OK, Stevenson DK. (1991) In vitro and in vivo characteristics of a heme oxygenase inhibitor: ZnBG. Am. J. Med. Sci. 302: 335-341.

21. Vreman HJ, Rodgers PA, Stevenson DK. (1990) Zinc protoporphyrin administration 
for suppression of increased bilirubin production by iatrogenic hemolysis in rhesus neonates. J. Pediatr. 117: 292.

22. Taraschi TF, Pouvelle B. (1994) There is no ducking the duct. Parasitol. Today 10: 212.

23. Lyberatos C, Chalevelakis G, Platis A, Stathakis N, Panani A, Gardikas C. (1972) Erythrocyte content of free protoporphyrin in thalassaemic syndromes. Acta Haematol. 47: 164.

24. Han P, Fung KP, Teo CP, Tam LP. (1990) Alpha-thalassaemia and beta-thalassaemia traits: Biological difference based on red cell indices and zinc protoporphyrin. Clin. Lab. Haematol. 12: 169.

25. Hill AV, Flint J, Weatherall DJ, Clegg JB. (1987) Alpha-thalassaemia and the malaria hypothesis. Acta Haematol. 78: 173.

26. Flint J, Hill AV, Bowden DK, et al. (1986) High frequencies of alpha-thalassaemia are the result of natural selection by malaria. Nature 321: 744.

27. Stevenson DK, Rodgers PA, Vreman HJ. (1989) The use of metalloporphyrins for the chemoprevention of neonatal jaundice. Am. J. Dis. Child 143: 353.

28. Valaes T, Petmezaki S, Henschke C, Drummond GS, Kappas A. (1994) Control of jaundice in preterm newborns by an inhibitor of bilirubin production: Studies with tin-mesoporphyrin. Pediatrics 93: 1.

29. Elford BC, Ferguson DJP. (1993) Secretory processes in Plasmodium. Parasitol. Today 9: 80-84.

30. Goldberg DE, Slater AFG, Beavis R, Chait B, Cerami A, Henderson GB. (1991) Hemoglobin degradation in the human malaria pathogen Plasmodium falciparum: A catabolic pathway initiated by a specific aspartic protease. J. Exp. Med. 173: 961.

31. Goldberg DE, Slater AFG, Cerami A, Henderson GB. (1990) Hemoglobin degradation

Contributed by A. Cerami on January 10, 1996. in the malaria parasite plasmodium falciparum: An ordered process in a unique organelle. Proc. Natl. Acad. Sci. U.S.A. 87: 2931.

32. Pouvelle B, Spiegel R, Hsiao L, et al. (1991) Direct access to serum macromolecules by intraerythrocytic malaria parasites. Nature 353: 73.

33. Sherman IW, Zidovetzki R. (1992) A parasitophorous duct in Plasmodium-infected red blood cells. Parasitol. Today 8: 2.

34. Cannon JB, Martin C, Drummond GS, Kappas A. (1993) Targeted delivery of a heme oxygenase inhibitor with a lyophilized liposomal tin mesoporphyrin formulation. Pharm. Res. 10: 715.

35. Luzzi GA, Torii M, Aikawa M, Pasvol G. (1990) Unrestricted growth of Plasmodium falciparum in microcytic erythrocytes in iron deficiency and thalassaemia. Br. J. Haematol. 74: 519-524.

36. Pasvol G, Wilson RJM. (1982) The interaction of malaria parasites with red blood cells. Br. Med. Bull. 38: 133.

37. Friedman MJ. (1979) Oxidant damage mediates variant red cell resistance to malaria. Nature 280: 245-247.

38. Brockelman CR, Wongsattayanont $B$, Tanariya P, Fucharoen S. (1987) Thalassemic erythrocytes inhibit in vitro growth of Plasmodium falciparum. J. Clin. Microbiol. 25: 56.

39. Nurse GT. (1979) Iron, the thalassemias, and malaria. Lancet Nov 3: 938-940.

40. Luzzi GA, Merry AH, Newbold CI, Marsh K, Pasvol G, Weatherall DJ. (1991) Surface antigen expression on Plasmodium falciparum-infected erythrocytes is modified in alpha- and beta-thalassemia. J. Exp. Med. 173: 785-791.

41. Carlson J, Nash GB, Gabutti V, Al-Yaman F, Wahlgren M. (1994) Natural protection against severe Plasmodium falciparum malaria due to impaired rosette formation. Blood. 84: 3909-3914. 